\title{
Evaluation of a strain based failure criterion for the multi-constituent composite model under shock loading
}

\author{
Christopher T. Key ${ }^{1, \text { a }}$, Shane C. Schumacher ${ }^{2}$, and C. Scott Alexander ${ }^{2}$ \\ ${ }^{1}$ HI-TEST Laboratories Inc., Applied Technologies Group, 32 Murphys Drive, Groton, CT 06340, USA \\ ${ }^{2}$ Sandia National Laboratories, PO Box 5800, Albuquerque, NM 87185, USA
}

\begin{abstract}
This study details and demonstrates a strain-based criterion for the prediction of polymer matrix composite material damage and failure under shock loading conditions. Shock loading conditions are characterized by high-speed impacts or explosive events that result in very high pressures in the materials involved. These material pressures can reach hundreds of kbar and often exceed the material strengths by several orders of magnitude. Researchers have shown that under these high pressures, composites exhibit significant increases in stiffness and strength. In this work we summarize modifications to a previous stress based interactive failure criterion based on the model initially proposed by Hashin, to include strain dependence. The failure criterion is combined with the multi-constituent composite constitutive model (MCM) within a shock physics hydrocode. The constitutive model allows for decomposition of the composite stress and strain fields into the individual phase averaged constituent level stress and strain fields, which are then applied to the failure criterion. Numerical simulations of a metallic sphere impacting carbon/epoxy composite plates at velocities up to $1000 \mathrm{~m} / \mathrm{s}$ are performed using both the stress and strain based criterion. These simulation results are compared to experimental tests to illustrate the advantages of a strain-based criterion in the shock environment.
\end{abstract}

\section{Introduction}

Composite materials are often used in shock loading applications caused by high-velocity impacts, where the strain rates can reach on the order of $10^{7}$ and the pressures are often in the hundreds of kbar. Hydrocodes are commonly used to simulate material response under such conditions. Hydrocodes typically separate a material's response into volumetric and deviatoric components as given in Eq. (1). The deviatoric $\left(s_{i j}\right)$ or distortional response of the material is modeled with a constitutive model, while the volumetric $(P)$ response is modeled using an equation of state (EOS).

$$
\sigma_{i j}=s_{i j}+P \delta_{i j}
$$

The work presented in this paper utilizes the shock physics hydrocode CTH [1] to model unidirectional composite materials under high velocity impacts where the pressures can reach very high levels. As a result of these high pressures, the pressure term on the right hand side of Eq. (1) can dominate the material's stress response. As a consequence, damage and failure predictions using traditional stress based failure criterion can often times be misleading. Factors which influence these misleading results include; stiffness and strength increases at elevated pressures [2], the retention of shearing resistance under compression and the fact that these materials often times cannot react on the time scales of the pressure wave propagations.

Figure 1 shows an example of the pressure dependent strength behavior for a graphite/epoxy composite material.

\footnotetext{
${ }^{a}$ Corresponding author: chris.key@hitestlabs.com
}

This data shows a nearly 2 times increase in the matrix dominated failure modes of the material as a result of the applied pressure being increased from standard atmospheric conditions to 6 kbar. Figure 1 also shows that fiber dominated failure modes are less influenced by the effect of pressure than are matrix modes. These observations lead to the suggestion that a deformation or strain-based criterion may be more suitable for shock loading problems.

In what follows, we briefly outline the composite material constitutive model and the equation of state model used for these initial studies. We then detail both the stress and strain-based failure criterion used along with the experimental test details used to support that analytical work. Finally, we compare the results of the two failure criterion against the experimental test results of a carbonepoxy panel subjected to spherical projectile impacts.

\section{Composite material models}

\subsection{Constitutive model}

To simulate the anisotropic response of a unidirectional composite material, the multi-constituent Composite Model (MCM) within the CTH constitutive material library was utilized. The MCM model is based on the work of Hill [3] and adaptation of Garnich and Hansen [4], where a detailed derivation of the approach and corresponding decomposition relationships can be found.

This MCM model is based upon a multicontinuum concept, which provides constituent (fiber and matrix) averaged stress and strain fields at the continuum level. To do this, the MCM model assumes that at each point 


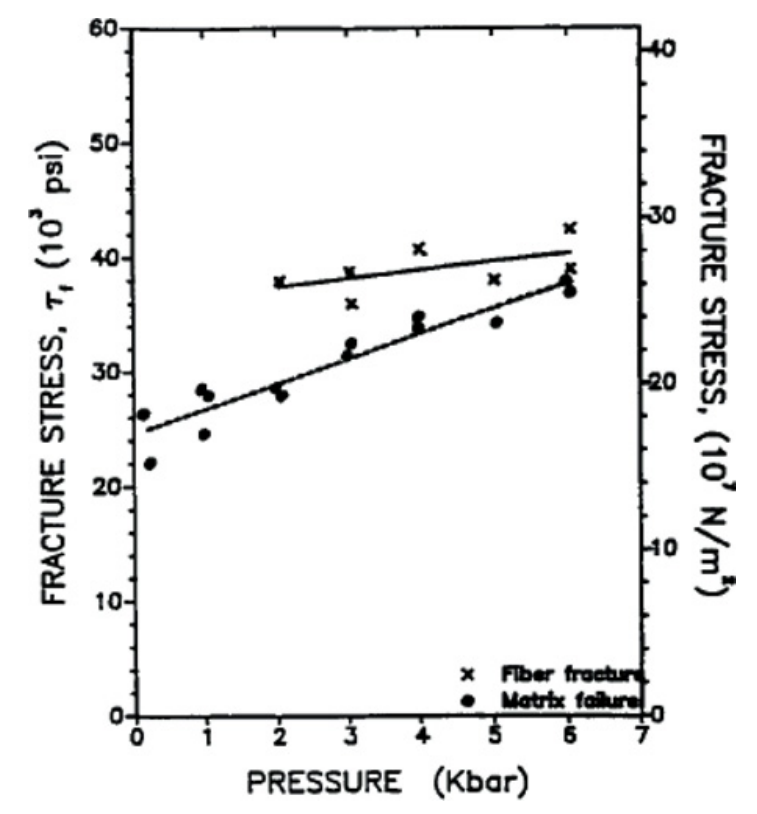

Figure 1. Pressure dependent strength data for fiber and matrix fracture modes in composite materials [2].

within a structure, there exists a representative volume element (RVE). For the unidirectional composite material considered in this work, Fig. 2 illustrates the hexagonally packed basic repeating unit microstructure used, where the red regions represent the carbon fibers and the blue regions are the epoxy matrix.

The volume averaged stress fields for each of the constituents, which comprise the RVE, can be expressed as:

$$
\bar{\sigma}_{i}=\frac{1}{V_{i}} \int_{D_{i}} \bar{\sigma}(X) d V .
$$

For the case of a unidirectional composite as shown in Fig. 2, $\mathrm{i}=2$, representing the fiber and matrix constituent.

Volume averaging leads to the relationship in Eq. (3) between the homogenized composite and constituent stress fields.

$$
\bar{\sigma}=\phi_{\text {matrix }} \bar{\sigma}_{\text {matrix }}+\phi_{\text {fiber }} \bar{\sigma}_{\text {fiber }} .
$$

Where in this equation $\phi_{\text {matrix }}$ and $\phi_{\text {fiber }}$ are the volume fractions of the matrix and fiber, respectively.

Combining Eqs. (2) and (3) with the linear elastic constitutive relationships for the composite and constituents, the following strain decomposition equation can be derived.

$$
\left\{\varepsilon_{\text {matrix }}\right\}=\left(\phi_{\text {matrix }}[I]+\phi_{\text {fiber }}[A]\right)^{-1}\{\varepsilon\}
$$

where,

$$
[A]=-\frac{\phi_{\text {matrix }}}{\phi_{\text {fiber }}}\left([C]-\left[C_{\text {fiber }}\right]\right)\left([C]-\left[C_{\text {matrix }}\right]\right) .
$$

Equation (4) provides the volume averaged continuum strain field in the matrix constituent as a function of the composite strain field $(\varepsilon)$ and the fiber $\left(C_{\text {fiber }}\right)$, matrix $\left(C_{\text {matrix }}\right)$ and composite $(C)$ stiffness matrices. Next, using the matrix averaged strain field, the fiber averaged strain

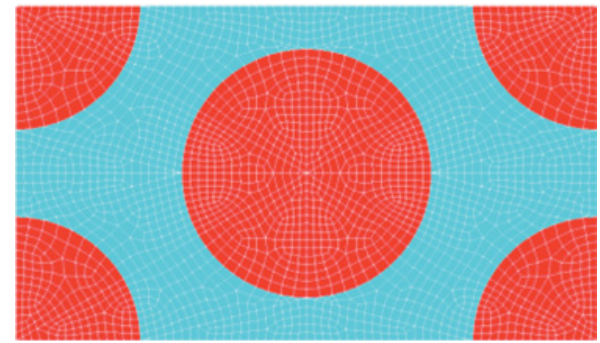

Figure 2. Hexagonally packed unidirectional composite microstructure.

field can be found by applying a similar volume averaging scheme as used in Eq. (3) to yield:

$$
\left\{\epsilon_{\text {fiber }}\right\}=\frac{1}{\phi_{\text {fiber }}}\left(\{\varepsilon\}-\phi_{\text {matrix }}\left\{\varepsilon_{\text {matrix }}\right\}\right) .
$$

Finally, the average stress fields for the fiber and matrix can be calculated using their respective linear elastic constitutive relationship.

\subsection{Equation of state coupling}

One implication of the anisotropy of the unidirectional composite material considered in this work, is the inherent coupling between the deviatoric and volumetric responses of the material. This coupling causes a problem in a hydrocode, where the deviatoric and volumetric responses are separated. Therefore, the MCM model must account for the fact that hydrostatic pressure applied to a finite volume causes not only a volume change, but also a shape or distortional change.

The coupling is accounted for within the MCM model using the relationships developed by Lukyanov [5]. Lukyanov's relationships modify the pressure term, which results from the EOS, such that an applied pressure only causes a volume change without a distortional change. This approach therefore retains the traditional definition of pressure.

Lukyanov expressed the pressure in term of a tensor rather than the traditional scalar according to Eq. (7).

$$
\tilde{P}=-\left(P^{*}\right) \alpha_{i j}
$$

Where $P^{*}$ is the scalar pressure defined as:

$$
P^{*}=P^{E O S}+\frac{\beta_{i j} s_{i j}}{\alpha_{i j} \beta_{i j}} .
$$

In Eq. (8), the scalar pressure is defined as the summation of the Mie-Grüneisen [6] EOS pressure $\left(P^{E O S}\right)$ and a coupling term which is a function of the deviatoric stress tensor $\left(s_{i j}\right)$. This coupling term is a function of the $\alpha_{i j}$ and $\beta_{i j}$ tensors, which are composed of the material stiffness and compliance, respectively. These coupling terms are formulated such that when combined with the deviatoric stress as shown in Eq. (8), the deviatoric strains are zero. The reader is referenced to Lukyanov [5] for a complete derivation and description of these terms, as the details are not included here for brevity. 
Finally, combining the relationship of Eq. (8) with the stress decomposition relationship of Eq. (1), Lukyanov arrives at the following relationship for the deviatoric stress:

$$
s_{i j}=\sigma_{i j}-\alpha_{i j} \cdot \frac{1}{\|\alpha\|} \sigma_{k l} \alpha_{k l} .
$$

Equation (9) accounts for the coupling effects of the pressure on the deviatoric stresses just as Eq. (8) accounted for the coupling effects of the deviatoric stresses on the pressure.

The reader is referenced to Schumacher and Key [7] for a detailed description of the anisotropic equation of state coupling model and its implementation within $\mathrm{CTH}$.

\subsection{Failure criteria}

The failure criteria used within the MCM model for this work is based on the unidirectional composite material failure criterion of Hashin [8]. Hashin's criterion predicted constituent level failure modes based on the stress fields of the homogenized composite. Mayes and Hansen [9] modified Hashin's criterion such that the fiber and matrix failure modes were predicted as a function of their individual volume averaged continuum stress fields, which are a direct result of the MCM model. Here we provide a brief review of the constituent level failure criteria of Mayes and Hansen. Further details of the development of these criterions can be found in Mayes [10] and Mayes and Hansen [9].

\subsubsection{Stress-based failure criterion}

The MCM criteria is based on the assumption that unidirectional fiber reinforced composite materials are transversely isotropic. Therefore, the criteria can be expressed in terms of transversely isotropic stress invariants [11] given in Eq. (10).

$$
\begin{gathered}
I_{1}=\sigma_{11}, \\
I_{2}=\sigma_{22}+\sigma_{33}, \\
I_{3}=\sigma_{22}^{2}+\sigma_{33}^{2}+2 \sigma_{23}^{2}, \\
I_{4}=\sigma_{12}^{2}+\sigma_{13}^{2}, \\
I_{5}=\sigma_{22} \sigma_{12}^{2}+\sigma_{33} \sigma_{13}^{2}+2 \sigma_{12} \sigma_{13} \sigma_{23} .
\end{gathered}
$$

Using the quadratic terms from Hashin's original criterion, the transversely isotropic stress invariants from Eq. (10) and simplifications detailed by Mayes and Hansen, the following general form of the MCM failure criterion is arrived at:

$$
A_{1} I_{1}^{2}+A_{2} I_{2}^{2}+A_{3} I_{3}+A_{4} I_{4}=1 .
$$

Where the $A$ terms are strength coefficients which are functions of the tensile, compressive and shear strength of the material.

Equation (11) can be simplified to predict matrix failure using the observation that conventional composite materials are composed of fibers which have significantly higher strengths than the matrix which bonds them together. Therefore, transverse failure of a unidirectional composite material is controlled by the strength of the matrix. If one assumes that the fibers of a unidirectional fiber reinforced composite run in the 1-direction, the $A_{1}$ coefficient in Eq. (11) can be set to zero, as it is only a function of the longitudinal stress $\left(\sigma_{11}\right)$ and the resulting matrix failure criterion used in the MCM model is then:

$$
{ }^{ \pm} A_{2}^{m}\left(I_{2}^{m}\right)^{2}+A_{3}^{m} I_{3}^{m}+A_{4}^{m} I_{4}^{m}=1 .
$$

The matrix strength coefficients in Eq. (12) are determined through application of in-plane shear, transverse shear, transverse tension and transverse compression uniaxial load cases resulting in the matrix failure strength coefficients give in Eq. (13).

$$
\begin{aligned}
{ }^{ \pm} A_{2}^{m} & =\frac{1}{\left({ }^{ \pm} S_{22}^{22 m}+{ }^{ \pm} S_{33}^{22 m}\right)^{2}} *\left(1-\frac{\left({ }^{ \pm} S_{22}^{22 m}\right)^{2}+\left({ }^{ \pm} S_{33}^{22 m}\right)^{2}}{2\left(S_{23}^{m}\right)^{2}}\right) \\
A_{3}^{m} & =\frac{1}{2\left(S_{23}^{m}\right)^{2}} \\
A_{4}^{m} & =\frac{1}{\left(S_{12}^{m}\right)^{2}} .
\end{aligned}
$$

In Eqs. (12) and (13) the \pm symbol indicates a dependence on the parameters for tensile vs. compressive stress.

Similar to the matrix failure criterion approach, one can assume that the higher strength of the fibers means the longitudinal failure of the composite material is controlled by the strength of the fibers. Hence, the $A_{2}$ and $A_{3}$ terms in Eq. (11) are set equal to zero as they are related to the transverse stresses $\left(\sigma_{22}\right.$ and $\left.\sigma_{33}\right)$. The resulting fiber failure criterion used in the MCM model is then:

$$
{ }^{ \pm} A_{1}^{f}\left(I_{1}^{f}\right)^{2}+A_{4}^{f} I_{4}^{f}=1 .
$$

The coefficients for the fiber failure criterion of Eq. (13) are determined by applied uniaxial loading conditions of in-plane shear, longitudinal compression and longitudinal tension, resulting in the fiber failure strength coefficients given in Eq. (15).

$$
\begin{aligned}
{ }^{ \pm} A_{1}^{f} & =\frac{1}{\left({ }^{ \pm} S_{11}^{f}\right)^{2}} \\
A_{4}^{f} & =\frac{1}{\left(S_{12}^{f}\right)^{2}} .
\end{aligned}
$$

\subsubsection{Strain-based failure criterion}

The strain-based failure criterion is identical in form to the stress-based failure criterion presented previously. The main difference being that the invariants are now the transversely isotropic strain invariants and the resulting strength variables $(S)$ in Eqs. (13) and (15) are the ultimate strain values of the composite material rather than the ultimate stress values. 


\section{Experimental testing details}

The high velocity impact experiments for this work were performed at Sandia National Laboratories using single and two stage light gas guns. The gas guns were used to fire $1 / 4$ inch diameter steel spheres at velocities from 250 to $1000 \mathrm{~m} / \mathrm{s}$. The test setup included flash X-ray or high-speed cameras to capture the penetration event, panel motion and projectile residual velocity if applicable. Following each impact test, the composite panel was inspected using ultrasound to measure the extent of damage that occurred during the impact event.

The composite material system used to fabricate the panel was IM7 carbon fibers pre-impregnated with a toughened epoxy (8552) from Hexcel. Each composite target panel was fabricated to be 7.5 inches by 7.5 inches square with a fiber volume fraction of $68 \%$ and thicknesses ranging from $1 / 4$ to 1 inch. The lamination schedule of each panel was quasi-isotropic $[0 / 90 /+45 /-45 /-45 /+45 / 90 / 0]_{a}$, with the repetitions of this base layup $(a)$ being repeated as necessary to achieve the overall desired panel thickness.

In this paper we focus only on two of the tests conducted which demonstrate the extremes of the composite damage. These two tests, impacting $1 / 2$ inch thick panels, include an impact velocity of $506 \mathrm{~m} / \mathrm{s}$ where the projectile did not penetrate the composite target panel and a $1073 \mathrm{~m} / \mathrm{s}$ impact where the projectile perforated the composite panel.

\section{Numerical model details}

The CTH numerical simulations used to evaluate the failure criteria where performed using a full 3-dimensional model of both the composite target panel and spherical projectile. The projectile was modeled using a simple rate-dependent plasticity constitutive model and a MieGrüneisen EOS with an initial density of $8.13 \mathrm{~g} / \mathrm{cm}^{3}$ to represent high-density steel.

The composite target panel was modeled using the previously described MCM composite model with a quasiisotropic layup and an initial density of $1.56 \mathrm{~g} / \mathrm{cm}^{3}$. The stiffness parameters for the fiber and matrix constituents were taken from Key et al. [12], while the composite stiffness properties were generated using the constituent properties and the finite element micromechanics model shown previously in Fig. 2. The initial constituent material strength properties were also determined from the micromechanics model and the composite material strength properties of Key et al. [12].

As a simple attempt to account for the strength increases that occur due to the elevated pressures and strains rates, a strength increase factor estimate of 1.75 was applied to each of the ultimate stress and strain properties. This factor was estimated from the data of Shin et al. of Fig. 1, where although the peak pressures in our problem are at or above $100 \mathrm{kbar}$, there was no experimental data found to support further strengthening above the $6 \mathrm{kbar}$ pressure of Shin.

Finally, each of the two impact velocity conditions was modeled using various different Eularian mesh densities as part of a larger effort. For this paper we will use the

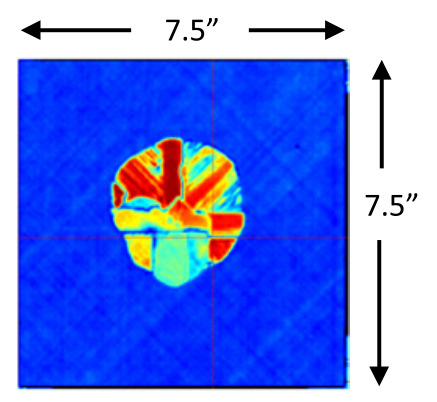

(a)

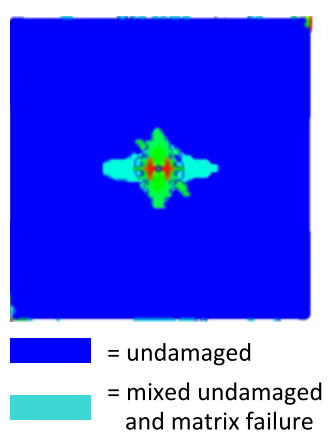

(b)

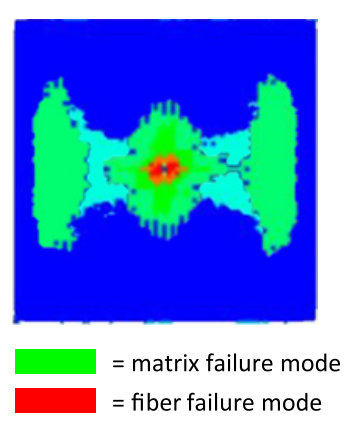

(c)
Figure 3. Damage patterns in (a) ultrasound data, (b) strain-based and, (c) stress-based predictions (Mesh 1) for $506 \mathrm{~m} / \mathrm{s} \mathrm{impact.}$

following naming convention when referring to each of the different densities:

$$
\begin{aligned}
& \text { Mesh 1: } 0.273 \mathrm{~cm} \times 0.273 \mathrm{~cm} \times 0.1524 \mathrm{~cm} \\
& \text { Mesh 2: } 0.136 \mathrm{~cm} \times 0.136 \mathrm{~cm} \times 0.0762 \mathrm{~cm} \\
& \text { Mesh 3: } 0.0683 \mathrm{~cm} \times 0.0653 \mathrm{~cm} \times 0.0635 \mathrm{~cm} \\
& \text { Mesh 4: } 0.0453 \mathrm{~cm} \times 0.453 \mathrm{~cm} \times 0.0423 \mathrm{~cm} .
\end{aligned}
$$

As part of a larger effort, the different mesh densities were chosen to study their effect on both the predicted damage patterns and the residual velocity predictions. However, the main focus of this paper is on the differences in the numerical predictions as related to the stress and strain-based failure criterion. Therefore, the mesh density differences are only provided in select results.

\section{Results}

In order to evaluate the performance of the MCM model using both the stress and strain-based failure criteria, two evaluation metrics were chosen. First, the damage patterns predicted by the analytical simulations were compared to the observed ultrasonic post-test patterns. Second, the measured projectile residual velocity from the experimental test was compared directly against the analytically predicted residual velocity.

It is noted here that in all subsequent numerical simulation figures, green indicates matrix failure modes such as matrix microcracking or delamination, while red indicates fiber failure modes. The colors in the ultrasound data indicate time of flight which is related to the depth of the damage region. 
Table 1. Residual velocity comparisons for $506 \mathrm{~m} / \mathrm{s}$ impact.

\begin{tabular}{|c|c|c|c|}
\hline & Mesh 1 & Mesh 2 & Mesh 3 \\
\hline Experimental & \multicolumn{3}{|c|}{$0 \mathrm{~m} / \mathrm{s}$} \\
\hline Strain-based criterion & $0 \mathrm{~m} / \mathrm{s}$ & $0 \mathrm{~m} / \mathrm{s}$ & $0 \mathrm{~m} / \mathrm{s}$ \\
\hline Stress-based criterion & $0 \mathrm{~m} / \mathrm{s}$ & $20 \mathrm{~m} / \mathrm{s}$ & $100 \mathrm{~m} / \mathrm{s}$ \\
\hline
\end{tabular}

\section{1. $1 / 2$ inch panel; $506 \mathrm{~m} / \mathrm{s}$ impact velocity}

Figure 3(a) shows the damage patterns measured from the ultrasonic inspection of the $1 / 2$ inch panel after the spherical projectile impacted the composite target panel at $506 \mathrm{~m} / \mathrm{s}$. From this image one can see that the damage envelope through the thickness of the target panel is nearly circular in shape and consists of striations, which align with the quasi-isotropic lamination schedule $\left(0^{\circ}, 90^{\circ}\right.$, $+45^{\circ}$ and $-45^{\circ}$ ) of the panel. The damage extent is also evident in this figure, as the entire 7.5 inch by 7.5 inch domain of the panel is shown.

Figure 3(b) shows the predicted damage envelope from the CTH-MCM simulation when using the strainbased failure criterion over the entire 7.5 inch by 7.5 inch panel domain. The damage envelope in this figure and all subsequent numerical images was generated by superimposing $2 \mathrm{D}$ damage slices of the laminate at the center of each ply. This approach was necessary as there is currently no capability available within the CTH post-processing package to generate the through thickness envelopes.

From Fig. 3(b) one can see that the strainbased criterion captures the basic characteristics of the damage envelope that are seen in the ultrasound results. Specifically, the predicted striations and extent of the damage in the $0^{\circ}$ direction (horizontal) is very similar to the extent in the ultrasound. The predicted damage extents or striation in the $90^{\circ},+45^{\circ}$ and $-45^{\circ}$ directions is also evident. However, the extent of these directions appears to be approximately $1 / 2$ the extent seen in the experimental data for the $90^{\circ},+45^{\circ}$ and $-45^{\circ}$ orientations.

Figure 3(c) shows the predicted results of the same simulation using the stress-based criterion. From this figure it is evident that the stress-based criterion significantly over-predicts the damage extent in the composite panel in the $0^{\circ}$ direction (horizontal) as compared to the ultrasound results. In this direction the damage is observed to extend nearly to the plate free-edge, with large regions of damage far from the central impact zone. Also, there is very little evidence of the directional striations seen in the experimental results and the strainbased results.

For this experimental configuration, the projectile was defeated by the composite panel such that the residual velocity of the projectile was $0 \mathrm{~m} / \mathrm{s}$. The residual velocities for the spherical projectile predicted by the CTH-MCM simulations are given in Table 1 for each of the failure criteria. The results in Table 1 show that the strain-based criterion predicts that the composite panel will defeat the spherical projectile, as was observed in the experimental testing, for each of the mesh densities. However, for the stress-based criterion, it is evident that as the mesh density increases, the model predicts that the projectile defeats the

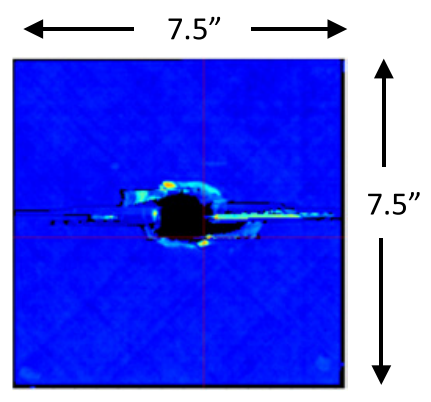

(a)

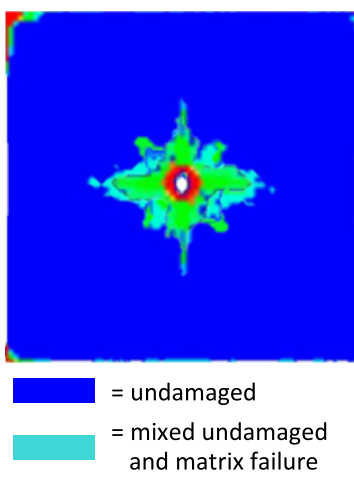

(b)

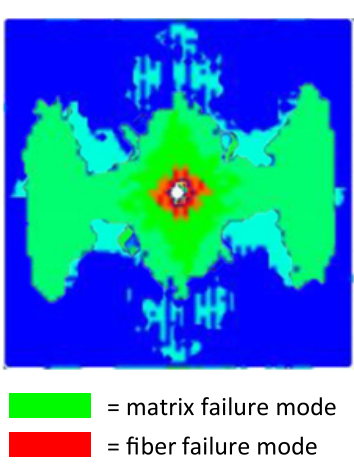

(c)
Figure 4. Damage patterns in (a) ultrasound data, (b) strain-based and, (c) stress-based predictions (Mesh 1) for $1076 \mathrm{~m} / \mathrm{s}$ impact.

composite panel and the residual velocity of the penetrator is approximately $100 \mathrm{~m} / \mathrm{s}$ for the highest mesh density.

Table 2. Residual velocity comparisons for $1076 \mathrm{~m} / \mathrm{s}$ impact.

\begin{tabular}{|l|c|c|c|c|}
\hline & Mesh 1 & Mesh 2 & Mesh 3 & Mesh 4 \\
\hline Experimental & \multicolumn{4}{|c|}{$562 \mathrm{~m} / \mathrm{s}$} \\
\hline $\begin{array}{l}\text { Strain-based } \\
\text { criterion }\end{array}$ & $25 \mathrm{~m} / \mathrm{s}$ & $300 \mathrm{~m} / \mathrm{s}$ & $525 \mathrm{~m} / \mathrm{s}$ & $560 \mathrm{~m} / \mathrm{s}$ \\
\hline $\begin{array}{l}\text { Stress-based } \\
\text { criterion }\end{array}$ & $150 \mathrm{~m} / \mathrm{s}$ & $325 \mathrm{~m} / \mathrm{s}$ & $450 \mathrm{~m} / \mathrm{s}$ & $500 \mathrm{~m} / \mathrm{s}$ \\
\hline
\end{tabular}

\section{2. $1 / 2$ inch panel; $1076 \mathrm{~m} / \mathrm{s}$ impact velocity}

Figure 4(a) shows the post-test ultrasound evaluation of the $1 / 2$ inch panel after being impacted at $1076 \mathrm{~m} / \mathrm{s}$. Unlike the slower velocity condition discussed in the previous section, under this increased velocity the projectile easily defeats the panel. As a result of the projectile penetrating the panel, the ultrasound image of Fig. 4(a) can be misleading. First, looking at the figure one might interpret the black regions as holes or perforations in the panel; however, this is not the case. The black regions in the ultrasound are regions on the panel where the ultrasound was unable to make a conclusive measurement due to the large and varying amounts of interference present in the ultrasound signal. Visual examination of the panel confirms that the perforation in the panel was almost exactly the same diameter as the spherical projectile ( $1 / 4$ inch).

Similar to the previous slower impact condition, the damage envelope predicted by the strain-based criterion 
(Fig. 4(b)) shows damage extents and patterns that are similar to those observed in the tested panel. The stressbased criterion results shown in Fig. 4(c) were again much greater in extent than what was observed in the experimental results. These prediction results were similar to the previous condition in that they extended nearly to the free-edges of the panel in the $0^{\circ}$ and $90^{\circ}$ directions.

Table 2 shows the residual velocity prediction for this impact velocity along with the experimentally measured residual velocity. These results show that both criterions are converging towards the experimental results. However, the strain-based criterion appears to be converging on the experimental result quicker than the stress-based criterion.

Tables 2 and 3 together also show that at the less dense mesh configurations (Mesh 1,2) a similar trend is observed where the stress-based failure criterion predicts a higher residual velocity. However, between Mesh 2 and Mesh 3, the trend reverses for the high velocity shot and the residual velocity for the strain-based criterion is higher than the stress-based residual velocity. Efforts are currently ongoing to better understand the mesh dependent behaviors seen in for each of these criterion results.

\section{Conclusions}

The preliminary results of this work show that a stressbased failure criterion tends to over predict the damage extent of the composite panels when subjected to shock loading. The results also show that this increased damage leads to an over-prediction of the projectile residual velocity. In contrast, the strain-based criterion appears to capture the basic characteristics of the damage patterns observed in the experimental testing and also does a good job of converging on the projectile residual velocity.
These results lead to the conclusion that when modeling composite materials under high velocity impact conditions, a strain based failure criterion may be more appropriate than a stress-based, pressure independent criterion. Future work will include the evaluation of a pressure-dependent, stress or strain-based criteria [13] to eliminate the artificial strengthening parameter applied during these early studies.

\section{References}

[1] J.M. McGlaun, S.L. Thompson, L.N. Kmetyk, M.G. Elrick, Int. J. Impact Eng. 10, 351 (1990)

[2] E.S. Shin, K.D. Pae, J. Compos. Mater. 26, 828 (1992)

[3] R. Hill, J. Mechan. Phys. Solids 12, 199-212 (1964)

[4] M.R. Garnich, A.C. Hansen, J. Compos. Mater. 31, 71 (1997)

[5] A.A. Lukyanov, Int. J. Plasticity 24, 140 (2008)

[6] E.S. Hertel, G.I. Kerley, Sandia National Laboratories Report SAND98-0947 (1998)

[7] S.C. Schumacher, C.T. Key, Sandia National Laboratories Report SAND2012-7714 (2012)

[8] Z. Hashin, J. Appl. Mech. 47, 329 (1980)

[9] J.S. Mayes, A.C. Hansen, Comp. Sci. Tech. 64, 379 (2004)

[10] J.S. Mayes, University of Wyoming Doctoral Dissertation (1999)

[11] A.C. Hansen, D.M. Blackketter, D.E. Walrath, J. Appl. Mech. 58, 881 (1991)

[12] C.T. Key, M.R. Garnich, A.C. Hansen, Compos. Struct. 65, 357 (2004)

[13] S.T. Pinho, P. Robinson, C Schuecker, P.P. Camanho, ICCM 17 Proceedings (2009) 